\title{
Análisis del estado de conservación del cerro Jánano, a partir del conocimiento de su mastofauna
}

\section{Analysis of the state of conservation of the Jánano Hill, based in knowledge of its mastofauna}

\author{
Nelsy Sofía Bonilla-Urrutia ${ }^{1}$, Luis Eladio Rentería Moreno²
}

\section{Resumen}

Este manuscrito contiene la caracterización de la mastofauna, registrada a lo largo del gradiente altitudinal del cerro Jánano, teniendo en cuenta la ausencia-presencia de grupos funcionales y especies relevantes (endémicas, amenazadas y susceptibles al tráfico), clave para el funcionamiento y conservación de este ecosistema, para lo cual se realizaron muestreos diarios (mañana y tarde), aplicando una evaluación rápida mediante observaciones directas e indicios, además de entrevistas informales a cazadores. Se registraron 29 especies, pertenecientes a 16 familias y 7 órdenes. Las familias más representativas fueron Didelphidae, Felidae y Procyonidae. Se obtuvieron importantes reportes como la alta representatividad de la especie dispersora Proechimys semispinosus y de Pecari tajacu, siendo esta última la especie con mayores registros (individuos y huellas) de presencia desde la falda hasta la cima del cerro. Se destacaron otros reportes importantes como los de Chironectes minimus y Leopardus wiedii que actúan como depredadores y controladores de otras poblaciones presa. También se comprobó la presencia de Alouatta palliata en cercanías al cerro, evidenciando la existencia de conectividad en el área. Se identificaron cuatro especies amenazadas y nueve con suceptibilidad al tráfico. Todas estas especies le confieren al ecosistema gran relevancia, corroborando su buen estado de conservación.

Palabras clave: Cerros del Chocó Biogeográfico, Estado de conservación, Mamíferos del Chocó, Serranía del Baudó.

\begin{abstract}
This manuscript contains the characterization of the mastofauna, registered along the altitudinal gradient of Cerro Jánano, taking into account the absence-presence of functional groups and relevant species (endemic, threatened and susceptible to
\end{abstract}

Bióloga, Estudiante de maestría en Ciencias Ambientales, Instituto de Investigaciones Ambientales del Pacífico. e-mail: nbonilla@iiap.org.co

2 Biólogo, Estudiante de maestría en Ciencias Biológicas, Instituto de Investigaciones Ambientales del Pacífico. e-mail: erenteria@iiap.org.co 


\section{Conservación y mastofauna. NS Bonilla-Urrutia, LE Rentería Moreno}

traffic), key to the operation and conservation of this ecosystem, for which daily samplings were carried out (morning and afternoon), applying a rapid evaluation through direct observations and indications, in addition to informal interviews with hunters. There were 29 species registered, belonging to 16 families and 7 orders. The most representative families were Didelphidae, Felidae and Procyonidae. Important reports were obtained such as the high representativeness of the dispersing species Proechimys semispinosus and Pecari tajacu, the latter being the species with the highest records (individuals and tracks) of presence from the skirt to the top of the hill. Other important reports were highlighted, such as those of Chironectes minimus and Leopardus wiedii that act as predators and controllers of other prey populations. The presence of Alouatta palliata near the hill was also proven, evidencing the existence of connectivity in the area. Four threatened species were identified and nine were susceptible to traffic. All these species give the ecosystem greatrelevance, corroborating its good state of conservation.

Keywords: Cerros del Chocó Biogeographic, Conservation status, Mammals of Chocó, Serranía del Baudó.

\section{Introducción}

La Serranía del Baudó es una emergencia desde la porción centro-occidental de Panamá hasta el norte del delta del río San Juan, en el Noroccidente de Colombia; se caracteriza por su aislamiento y por pertenecer a la reciente formación orogénica del Baudó. Esta articulación entre los dos continentes sur y norte se ha convertido, a través del tiempo, en el sitio de intercambio entre la biota de Sur, Centro y Norte América y viceversa. Sumado a esta extraordinaria ubicación geográfica, la Serranía del Baudó se encuentra inmersa dentro de la región con los regímenes de pluviosidad más altos del planeta, lo cual favorece a un sinnúmero de formas de vidas y hábitos de crecimiento, que alcanzan su máxima expresión de riqueza en estas zonas. Son estas características las que han facilitado el desarrollo de múltiples procesos de especiación y con ello la aparición de una amplia diversidad faunística, que juega un papel primordial en los procesos ecológicos de tan importante zona; es un elemento primordial para el mantenimiento de la biodiversidad, gracias a su constante interacción con la biota asociada con estos ambientes, que al ser conservados, se convierten en el refugio de una variada gama de especies animales. Además, esta fauna cumple funciones ecológicas propias de cada especie, con lo cual contribuye con el mantenimiento y balance de la dinámica ecosistémica del lugar. Sin embargo, muchas poblaciones han sido diezmadas y necesitan de evaluaciones constantes de sus densidades, para poder influir sobre las decisiones estratégicas que garanticen su presencia para las generaciones futuras.

Por lo anterior, mediante esta investigación, se pretendió estudiar el potencial mastofaunístico del cerro Jánano, partiendo del conocimiento de su composición y de la identificación de especies relevantes (grupos funcionales, especies de alta movilidad, endémicas, amenazadas y susceptibles al tráfico), claves para el funcionamiento y conservación de este ecosistema, de manera que la información recopilada y científicamente analizada sea útil para la toma de decisiones en términos de manejo ambiental de este tipo de ecosistemas. Lo anterior hace preguntar si la mastofauna presente en el cerro Jánano es un determinante ecológico de su buen estado de conservación.

Area de estudio. El cerro Jánano se encuentra localizado en el municipio de Nuquí y constituye una extensión de la serranía del Baudó, alcanzando una elevación de $631 \mathrm{msnm}$. Esta serranía compone un espinazo asimétrico, con pendientes suaves hacia el oriente, donde afloran sedimentos del terciario inferior y con taludes muy empina- 
dos hacia el occidente, muy difíciles de transitar, donde se presenta basaltos-cretácicos terciarios; la máxima altura con $1.810 \mathrm{msnm}$, se alcanzan en el Alto del Buey, al suroccidente de Bahía Solano. Las corrientes que van al occidente son cortas y a su entrada al océano forman escasas y exiguas playas, en una costa plena de acantilados que semejan mucho la costa rocosa del sur de Chile (Leyva 1993).

La serranía del Baudó se prolonga desde la parte centro occidental de Panamá y termina, luego de casi $350 \mathrm{~km}$, al norte del delta del río San Juan; fuera del departamento se prolonga bajo el fondo del océano reapareciendo en las islas Gorgona y Gorgonilla y en el departamento de Nariño, en la serranía del Remolino. La serranía del Baudó presenta un fuerte fracturamiento en dirección norte-sur (MMA-IGAC 2000),

Descripción del área de muestreo. El área de muestro correspondió a un mosaico paisajístico incluido dentro de un gradiente altitudinal, que va desde los 0 hasta los $631 \mathrm{msnm}$; a nivel general comprende un bosque maduro, donde no se identificaron intervenciones antrópicas importantes que pudieran alterar significativamente la sana dinámica trófica de los ecosistemas existente en el área, evidenciándose por el contrario un gran potencial para la ocurrencia de especies faunísticas de comportamiento crítico, raras o asociadas con hábitats maduros o relictuales.

El área de muestreo se caracterizó por presentar distintas estructuras vegetales que variaron a medida que los muestreos se enfocaban en la parte alta o baja del cerro Jánano, de allí que, en la zona correspondiente a la base del cerro, se identificó una importante presencia de cuerpos de agua, que proporcionaban gran humedad al sotobosque, la cual también se vio favorecida por la presencia de un dosel cerrado que limitaba en parte el paso de los rayos solares. En estas áreas el sotobosque era despejado y el suelo presentaba gran cantidad de hojarasca y tronco de árboles caídos que se establecen como refugios potenciales e ideales para pequeños y medianos vertebrados; la arquitectura y estructura vegetal estuvo representada principalmente por un nivel arbustivo y arbóreo de 3 a $25 \mathrm{~m}$ de altura (Figura 1), con una importante presencia de palmas, que proporcionan alimento y sustrato para aquellas especies de mamíferos de comportamientos arborícolas o que incluyen este recurso en sus comportamientos tróficos.

También se identificó que a medida que au-

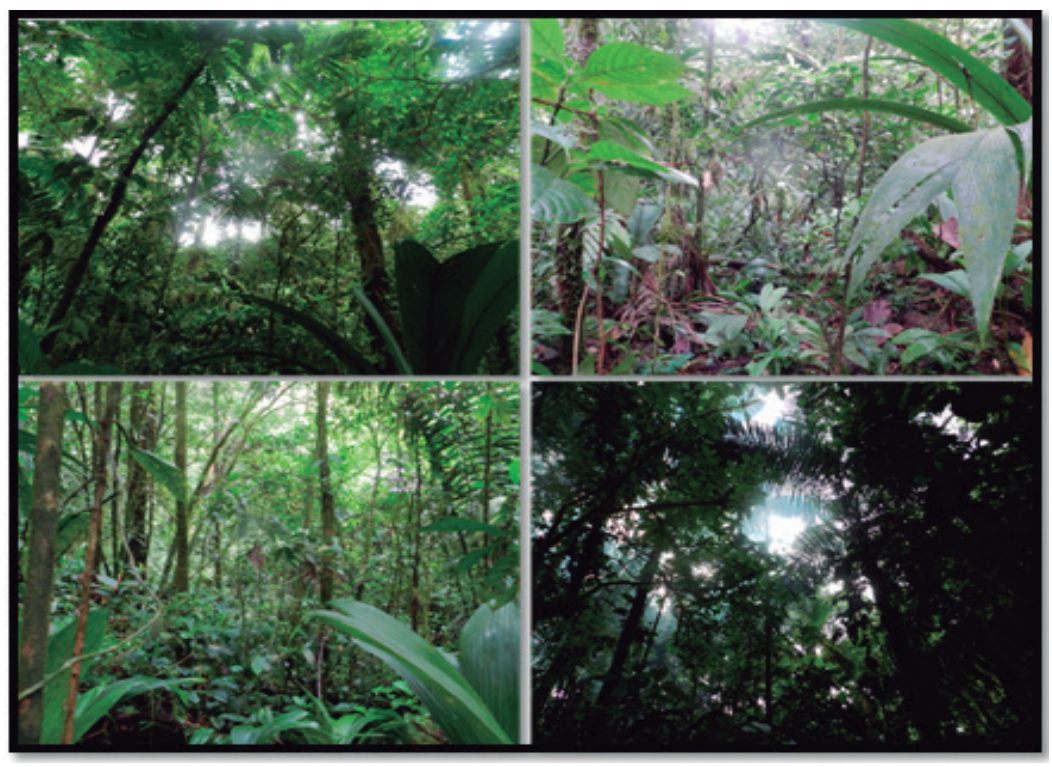

Figura 1. Características del área de estudio. 
menta el gradiente del cerro, estas características sufren algunas modificaciones, representadas por la ausencia de cuerpos de agua, una vegetación más baja con un estrato arbóreo y arbustivo entre uno y 15 metros y pendiente mucho más pronunciada, hechos que podrían limitar la ocurrencia y movilidad para algunos mamíferos. La cima se caracterizó por un suelo cubierto con abundante musgo que proporciona una excesiva humedad al sotobosque y pocos árboles muy epifitados y con poco follaje, dando la sensación de un pequeño bosque de niebla.

\section{Métodos}

Para lograr los objetivos de esta investigación, se aplicaron muestreos ecológicos rápidos (MER) (Sobrevila 1992), para lo cual se hicieron recorridos con el fin de obtener registros directos e indirectos. Estos recorridos consistieron en la búsqueda de individuos y de indicios (cuevas, huellas, sobras de alimento, comederos), que indicaran la presencia de especies en el lugar; los indicios encontrados fueron fotografiados. Los puntos de muestreo se seleccionaron teniendo en cuenta las características heterogéneas de los diferentes órdenes de mamíferos según lo propuesto por Rodríguez (1987) y Tirira (2000). Los muestreos se concentraron en la falda del cerro, debido a lo difícil del acceso a lo largo del gradiente. La identificación de las especies se realizó mediante las guías propuestas por Emmons (1999) y Navarro y Muñoz (2000). También se realizaron entrevistas informales a cazadores, quienes ayudaron a identificar, con la utilización de guías ilustradas, la existencia de especies de mamíferos en el lugar; estos además hicieron acompañamiento durante los recorridos, corroborando la identificación de indicios.

El estado de conservación o nivel de amenaza de los mamíferos que habitan el cerro Jánano se analizó basado en las listas Rojas de la UICN (2013), que menciona las diferentes categorías de amenaza, de las cuales solo se tuvieron en cuenta las siguientes, por presentar niveles de riesgo relevantes para la conservación: En Peligro Crítico(CR), En Peligro(EN) y Vulnerable(VU). También se analizó la información de las especies que aparecen listadas en el CITES.

\section{Resultados}

Mamíferos presentes en el cerro Jánano. Se registraron 29 especies, pertenecientes a 16 familias y 7 órdenes. Estas especies fueron reportadas por cazadores habitantes del área de influencia mediante entrevistas; sin embargo, 12 de las mismas fueron registradas en el área por medio de observaciones directas e indicios (huellas, cuevas y comederos), confirmando su presencia en el lugar (Tabla 1).

Esta diversidad se atribuye a varios factores como el tipo de formación orogénica, la heterogeneidad del paisaje, la variada y abundante oferta de recursos tanto tróficos como habitacionales y por la potencial conectividad paisajística de este con otros gradientes de la Serranía, además, de las fuentes hídricas existentes en la falda del cerro (quebrada Agua Bonita), la cual favorece la presencia de variados mamíferos terrestres y con hábitos semiacuáticos. Estas características biofísicas permiten destacar este cerro como un área prístina, el cual posiblemente ha mantenido las propiedades en buen estado en el tiempo y el espacio.

Presencia de especies clave. La presencia de carnívoros en el área se atribuye a la abundancia y variedad de especies presa potenciales en el lugar, como en el caso de pequeños roedores (ratones), frecuentemente observados durante los muestreos. Durante los recorridos se registró la presencia de un tigrillo (L. wiedii) observado en la ribera del río, en horario diurno. Según Oliveira (1998) y Domínguez y Ceballos (2005), a pesar de la amplia distribución de esta especie, es muy raro observarla en vida silvestre, sobre todo du- 
Bioetnia Volumen 13, 2016

Tabla 1. Composición de la mastofauna presente en el cerro Jánano

\begin{tabular}{|c|c|c|c|}
\hline Orden & Familia & Especie & Nombre común \\
\hline \multirow{4}{*}{ Didelphimorphia } & Didelphidae & Philander opossum & Cuatro ojos \\
\hline & & Didelphis marsupialis & Chucha \\
\hline & & Chironectes minimus & Chucha de agua \\
\hline & & Caluromys philander & Chuchita \\
\hline \multirow{4}{*}{ Rodentia } & Sciuridae & Sciurus granatensis & Ardilla \\
\hline & Echimyidae & Proechimys semispinosus & Ratón de monte \\
\hline & & Hoplomys gimnurus & Ratón espinoso \\
\hline & Cuniculidae & Cuniculus paca & Guagua \\
\hline \multirow{4}{*}{ Cingulata } & Dasyproctidae & Dasyprocta punctata & Guatín \\
\hline & Erethizontidae & Coendou sp. & Puerco espín \\
\hline & Dasypodidae & Dasypus novemcinctus & Armadillo \\
\hline & & Cabassous sp. & Armadillo \\
\hline \multirow[t]{2}{*}{ Artiodactyla } & Tayassuidae & Pecari tajacu & Tatabro \\
\hline & Cervidae & Mazama temama & Venado \\
\hline \multirow{3}{*}{ Pilosa } & Bradypodidae & Bradypus variegatus & Perezoso tres dedos \\
\hline & & Choloepus hoffmanni & Perezoso dos dedos \\
\hline & Myrmecophagidae & Tamandua maxicana & Oso hormiguero \\
\hline \multirow{3}{*}{ Primates } & Cebidae & Cebus capucinus & Mono capuchino \\
\hline & & Alouatta palliata & Mono aullador negro \\
\hline & Callitrichidae & Saguinus geoffroyi & Mono tití \\
\hline \multirow{9}{*}{ Carnívora } & Procyonidae & Potos flavus & Cuzumbí \\
\hline & & Nasua nasua & Cuzumbo \\
\hline & & Procyon cancrivorus & Mapache cangrejero \\
\hline & Felidae & Panthera onca & Tigre \\
\hline & & Leopardus wiedii & Tigrillo \\
\hline & & Leopardus tigrinus & Tigrillo \\
\hline & & Puma concolor & León \\
\hline & Mustelidae & Eira barbara & Zorro \\
\hline & & Lontra longicaudis & Nutria \\
\hline
\end{tabular}

rante el día, porque presenta hábitos nocturnos; su registro directo es un dato muy relevante para esta investigación; adicionalmente Bianchi et al. (2011) argumentan que esta especie presenta gran preferencia por los marsupiales y roedores, lo cual relaciona su presencia con la alta oferta de ratones y chuchas de agua en el lugar.

Durante el estudio se registraron 5 individuos de C. minimus observados en horario nocturno, sumergidos en los cuerpos de agua, los cuales al detectar la presencia humana, emergían y huían hacia tierra firme. Al respecto, Voss y Emmons (1996) afirman que, debido a sus hábitos semiacuáticos y a su baja tasa poblacional, esta especie es difícilmente observada en campo y excepcionalmente en ocasiones, se le puede observar recorriendo arroyos, siguiendo senderos de cursos de agua o en el borde del agua; y agregan que por esta razón, es poco lo que se conoce de su biología y ecología. Sin embargo, el hecho de haber observado este número de individuos en un MER durante este estudio, indica la ocurrencia de una 
población potencialmente abundante en el área. Además, C. minimus es un marsupial carnívoro que consume desde peces y crustáceos hasta ranas (Marshall 1978), actuando como controlador de diversas poblaciones de especies ícticas y anfibias. Esta especie además indica la presencia de abundante recurso trófico en el lugar, al tiempo que pone en evidencia interacciones ecológicas complejas, contribuyendo con la estabilidad y buena dinámica del ecosistema.

La presencia de este grupo funcional en el lugar es un indicador de la buena salud de este ecosistema, pues pone en evidencia la ocurrencia de procesos ecológicos como la depredación, porque los individuos de este grupo actúan como controladores biológicos de otras poblaciones de invertebrados y vertebrados, inhibiendo casos de sobrepoblación de especies muy prolíferas, al tiempo que ayudan en la transformación de la energía y la materia en los últimos niveles de las cadenas alimenticias de este ecosistema, indicando a la vez, la complejidad de las redes tróficas ocurrentes en la zona; esta afirmación es corroborada por el MAVDT y CIC (2010) quienes argumentan que las especies de este grupo tienen la función de actuar como agentes limitantes en el crecimiento de las poblaciones de otros animales, regulando y dinamizando con ello la energía que fluye a través de los ecosistemas, representando un eslabón clave en el buen funcionamiento y mantenimiento de los mismos.

La ocurrencia notable de roedores en el área se ve favorecida en primer lugar, por la heterogeneidad de microhábitats, así como de elementos (árboles, troncos caídos, raíces de grandes árboles y hojarasca) que permiten la construcción de cuevas, galerías y refugios temporales, los cuales proporcionan protección y facilitan el desarrollo de procesos vitales de las especies. En segundo lugar, a la disponibilidad de recursos tróficos presentes en el sitio, como árboles frutales, palmas, invertebrados y pequeños vertebrados, que se constituyen en factores que favorecen la abundancia de estos grupos en el lugar. Por ejemplo, en el área, se encontraron muchos ratones de monte en horario nocturno, poniendo en evidencia la ocurrencia de importantes abundancias de especies dispersoras de semillas, que contribuyen notoriamente con la formación y mantenimiento de la estructura vegetal, lo que se traduce en una alta capacidad que gracias a estas especies, tienen los bosques para regenerarse y mantenerse continuos en el tiempo y el espacio.

P. tajacu "tatabro" presentó registros de indicios (huellas y plantas forrageadas) desde la falda hasta la cima del cerro(Figura2), que evidenciaron la utilización del gradiente como un corredor de tránsito para esta especie, la cual posiblemente encuentra las condiciones ideales para desarrollar procesos de nutrición, reproducción, movilidad y

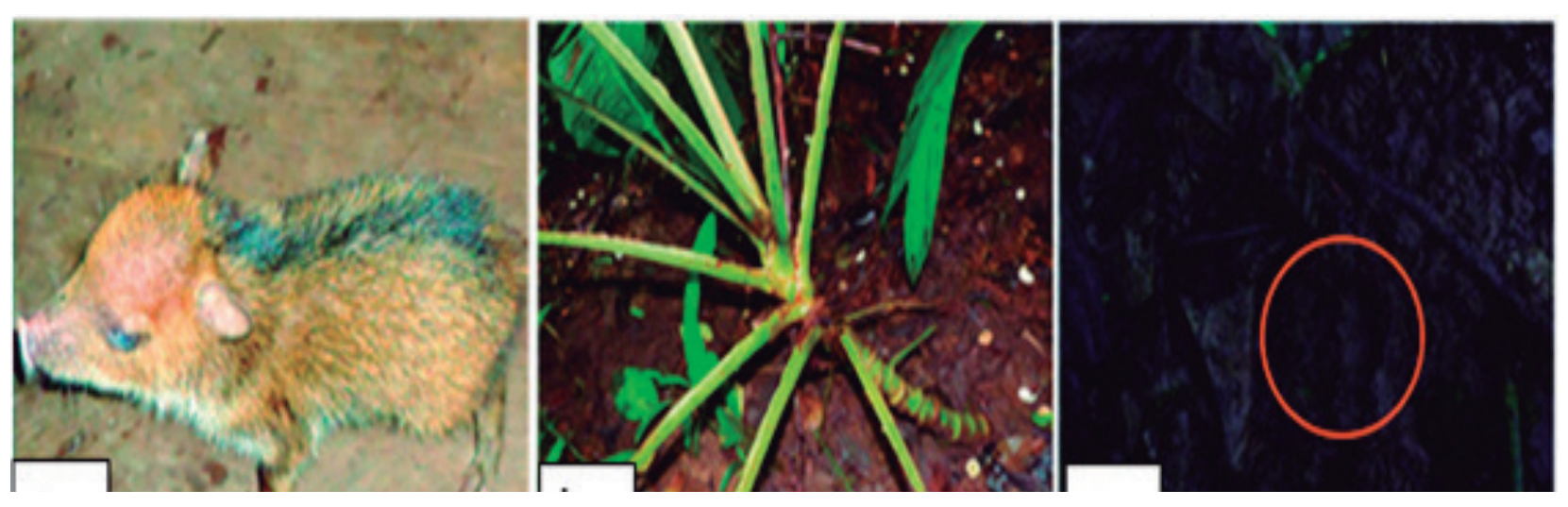

Figura 2. Indicios de la presencia de $P$. tajacu en el cerro Jánano; $\mathrm{a}=$ individuo, $\mathrm{b}=$ planta mordida y $\mathrm{c}=$ huellas. 


\section{Bioetnia Volumen 13, 2016}

descanso en el lugar. Al respecto Bello-Gutiérrez et al. (2005) argumentan que los sitios que habita esta especie tienen la mayor altitud, pendiente y densidad de arbustos; además, mencionan que el pecarí de collar se adapta a variados tipos de vegetación siempre y cuando cuenten con áreas amplias; mientras Bello (1993) por su parte, afirma que áreas con cubierta densa formada por lianas, bejucos y algunos arbustos, aún con poca superficie de extensión, pueden ser ocupados por el pecarí de collar como lugares de descanso y protección. Lo anterior permite deducir que, pese a que esta especie tolera sitios con diferentes estados de conservación, entre ellos áreas con alteraciones antrópicas importantes, este registro indica la buena funcionalidad del cerro en la totalidad de su gradiente y la ocurrencia de conectividad con otros puntos estratégicos de la serranía, lo cual favorece la buena integridad ecológica del ecosistema.

De otra parte, la presencia de $P$. tajacu en la zona, también se ve favorecida por la ocurrencia de abundantes individuos de Rhodospatha of moritziana (Araceae) y Cyclanthus sp. (Ciclantaceae), pues se registró, desde la falda del cerro hasta aproximadamentelos $200 \mathrm{~m}$, un considerable número de individuos con indicios de herbivoria y huellas de P. tajacu alrededor de los mismos, en especial $R$. moritziana, por lo cual se considera a esta herbácea como el elemento principal de la dieta de este mamífero en el lugar. Sin embargo, mediante estudios realizados sobre la dieta del pecarí de collar, algunos autores como Martínez y Mandujano (1995) y Pérez y Reyna (2008), concluyen que el principal elemento de su dieta lo constituyen los frutos, encontrando muestras de estas estructuras mordidas por la especie, lo cual difiere de la presente investigación, pues no se registraron sobras de frutos que indicaran tal comportamiento.

Durante los muestreos se percibieron vocalizaciones de $A$. palliata "mono aullador negro", lo que pone en evidencia la existencia de poblaciones en el área de influencia del cerro Jánano. La presencia de esta especie indica la ocurrencia de vegetación arbórea continua, lo que implica sucesos de conectividad ecológica para esta y otras especies de primates en la zona, pues según Defler(2003)y Gómez et al. (2010), los aulladores prefieren los estratos superiores del bosque, con buena continuidad de dosel y árboles de gran porte, así como del buen estado de este ecosistema. Por otro lado, autores como Julliot (1996) y Bravo y Sallenave (2003) han reportado la importancia del papel de $A$. palliata como dispersor de semillas, por lo que su presencia en este ecosistema implica procesos ecológicos tan relevantes para el mantenimiento de la conectividad y funcionalidad de este ambiente, como es la dispersión.

\section{Poblaciones importantes de especies amena-} zadas. Entre los registros obtenidos en este estudio, se identificaron cuatro especies ubicadas por la UICN dentro de la categoría de vulnerable (VU) (UICN 2013) y nueve están incluidas dentro de los apéndices I y II del CITES (2012) (Tabla 2).

La presencia de $P$. tajacu a lo largo del gradiente del cerro Jánano cobra mayor relevancia al destacarla como una especie susceptible al tráfico ilegal, situación que ha llegado a amenazar sus poblaciones a nivel nacional. Sin embargo, el hecho de encontrar una población importante de esta especie en el área, así como de albergar un número considerable de especies amenazadas, hacen de Jánano un área de gran relevancia para la conservación.

En cuanto a las actividades antrópicas, la cacería es la única que se realiza en el lugar. Esta es practicada de manera ocasional por algunos cazadores indígenas habitantes de comunidades adyacentes, quienes, por la lejanía del lugar, lo visitan muy esporádicamente, por lo que se infiere que actualmente las poblaciones de mamíferos de este lugar no sufren presión significativa, corroborando su significativo nivel de conservación. 
Conservación y mastofauna. NS Bonilla-Urrutia, LE Rentería Moreno

Tabla 2. Especies amenazadas en el cerro Jánano, Nuquí

\begin{tabular}{|c|c|c|c|c|}
\hline \multirow[t]{2}{*}{ Orden } & \multirow[t]{2}{*}{ Familia } & \multirow[t]{2}{*}{ Especie } & \multicolumn{2}{|c|}{ Amenazas } \\
\hline & & & UICN & CITES \\
\hline Artiodactyla & Tayassuidae & Pecari tajacu & & II \\
\hline \multirow{3}{*}{ Pilosa } & Bradypodidae & Bradypus variegatus & & II \\
\hline & Myrmecophagidae & Tamandua maxicana & VU & \\
\hline & & Alouatta palliata & VU & 1 \\
\hline Primates & Callitrichidae & Saguinus geoffroyi & & 1 \\
\hline \multirow{5}{*}{ Carnívora } & Felidae & Panthera onca & VU & 1 \\
\hline & & Leopardus wiedii & & I \\
\hline & & Leopardus pardalis & & 1 \\
\hline & & Puma concolor & & 1 \\
\hline & & Lontra longicaudis & VU & I \\
\hline
\end{tabular}

\section{Conclusiones}

De acuerdo con los resultados obtenidos, se puede inferir que el cerro Jánano presenta una mastofauna de gran relevancia ecológica para su funcionalidad, sostenibilidad e integridad, contando con una composición propia de ecosistemas saludables y originales, que pueden ser denominados como sitios estratégicos donde se conserva la vida en sus diferentes formas y donde concurre una notable biodiversidad. Al tiempo, es importante resaltar la presencia de mamíferos clave, de alta importancia ecológica, que favorecen la ocurrencia de una amplia gama de procesos ecológicos como la conectividad ecológica, dispersión de propágulos, depredación y control de poblaciones, transformación de la energía y la materia, transferencia genética, entre otras. Pero también se resalta la presencia de especies con gran riesgo ante procesos de extinción a nivel nacional y cuyas poblaciones en otras localidades se encuentran amenazadas por actividades antrópicas; todas estas especies le confieren al ecosistema gran relevancia, corroborando su buen estado de conservación.

\section{Literatura citada}

Bello J. 1993. Situación actual del orden Artiodactyla en la región de los Tuxtlas, Veracruz. Tesis de licenciatura. Xalapa: Universidad Veracruzana.

Bello-Gutiérrez J, Guzmán-Aguirre C, Chablé-Montero C. 2005. Caracterización del hábitat de tres especies de artiodáctilos en un área fragmentada de Tabasco, México. Memorias: Manejo de Fauna silvestre en Amazonia y Latinoamérica. URL disponible en: http://www. comfauna.org/wp-content/uploads/2012/PDFs-Manejofaunasilvestre/Iquitos-2004/3 Investigacion_Biologica_Aplicable_al_Manejo/136-145 jbello_CaracterizacionHabitatTresEspeciesArtiodactilos.pdf

Bianchi de RdeC, Rosa AF, Gatti A, Mendes SL. 2011. Diet of margay, Leopardus wiedii, and jaguarundi, Puma yagouaroundi (Carnivora: Felidae) in Atlantic Rainforest, Brazil. Zoologia. 28 (1): 127-32. URL disponible en: http://www.scielo.br/scielo.php?scrip$\mathrm{t}=\mathrm{sci}$ arttext\&pid=S1984-46702011000100018

Bravo SP, Sallenave A. 2003. Foraging behavior and activity patterns of Alouatta caraya in the northeastern Argentinean flooded forest. Internat J Primatol. 24: 825-46. URL disponible en: https://link.springer.com/ article/10.1023/A:1024680806342

CITES y UNEP. 2010. Convención sobre el Comercio Internacional de Especies Amenazadas. Apéndices I, II y III. URL disponible en: http:// www.cites.org

Convención sobre el Comercio Internacional de Especies Amenazadas (CITES). 2012. Apéndices I, IIy III. URL 


\section{Bioetnia Volumen 13, 2016}

disponible en: http://www.cites.org

Defler TR. 2003. Primates de Colombia. En: Rodríguez JV (ed.). Serie de guías tropicales de Colombia 4. Bogotá: Conservación Internacional.

Domínguez-Castellanos Y, Ceballos G. 2005. Un registro notable del tigrillo (Leopardus wiedii) en la Reserva de la Biosfera Chamela Cuixmala, Jalisco. Rev Mex Mastozool. 9: 146-9. URL disponible en: http://www. ecologia.unam.mx/laboratorios/eycfs/faunos/art/Gce/ AA53.pdf

Emmons LH. 1999. Mamíferos de los bosques húmedos de América Tropical. Una Guía de Campo. Santa Cruz de la Sierra: Editorial Fundación Amigos de la Naturaleza; 298 pp.

Gómez-Posada C, Giraldo PC, Álvarez Z. 2010. Evaluación de las poblaciones de mono aullador (Alouatta seniculus) en dos localidades en Quindío y Risaralda, Colombia. En: Pereira-Bengoa V, Stevenson PR, Bueno ML, Nassar-Montoya F (eds.). Primatología en Colombia: avances al principio del milenio. Bogotá: Fundación Universitaria San Martín.

Julliot C. 1996. Seed dispersal by red howling monkeys (Alouatta seniculus) in the tropical rain forest of French Guiana. Internat J Primatol. 17 (2): 239-58. URL disponible en: https://link.springer.com/article/10.1007/ BF02735451

Leyva P. 1993. Colombia Pacífico. Tomo I. Bogotá: Fondo FEN; 872 pp.

Marshall LG. 1978. Chironectes minimus. Am Soc Mammal Mammalian Sp. 109: 1-6.

Martínez-Romero LE, Mandujano S. 1995. Hábitos alimentarios del pecarí de collar (Pecari tajacu) en un bosque tropical caducifolio de Jalisco, México. Acta Zool Mex. 64: 1-20. URL disponible en: http://www. redalyc.org/articulo.oa? $\mathrm{id}=57506401$

MMA-IGAC. 2000. Zonificación ecológica de la región pacífica colombiana. Bogotá: Ministerio del Medio Ambiente e Instituto Geográfico Agustín Codazzi.

Ministerio de Ambiente, Vivienda y Desarrollo Territorial y Conservación Internacional Colombia. 2010. Guía de campo para la conservación de los felinos del Caribe colombiano. Felinos del Caribe: identidad biológica y cultural en una ecoregión felina. Bogotá: MAVDT, CIC; 20 pp. URL disponible en: http://www.academia.
edu/5860944/Gu\%C3\%ADa de campo para la conservaci $\% \mathrm{C} 3 \% \mathrm{~B} 3 \mathrm{n}$ de los felinos del Caribe colombiano Felinos del Caribe identidad biol $\% \mathrm{C} 3 \% \mathrm{~B}$ 3 gica_y_cultural en una ecorregi $\% \mathrm{C} 3 \% \mathrm{~B} 3 \mathrm{n}$ felina

Navarro JF, Muñoz J. 2000. Manual de huellas de algunos mamíferos terrestres de Colombia. Medellín: Edición de Campo. URL disponible en: https://rastreocolombia.jimdo.com/guias/manual-de-huellas-de-algunos-mam $\% \mathrm{C} 3 \%$ ADferos-terrestres-de-colombia/

Oliveira TG de. 1998. Leopardus wiedii. Mammalian Species. 579: 1-6. URL disponible en: http://www.bio-nica. info/Mammalia/Leopardus_wiedii.pdf

Pérez-Cortéz S, Reyna-Hurtado R. 2008. La dieta de los pecaríes (Pecari tajacu y Tayassu pecari) en la región de Calakmul, Campeche, México. Rev Mex Mastozool. 12: 17-42. URL disponible en: http://www.academia. edu/6215397/LA DIETA DE LOS PECAR\%C3\%8DES Pecari tajacu y Tayassu pecari EN LA REGI\%C3\%93N DE CALAKMUL CAMPECHE M\%C3\%89XICO

Rodríguez TM. 1987. Aporte al conocimiento de los buprestidos de Chile (Coleoptera: Buprestidae). Cuarta contribución. Rev Chil Entomol. 15: 13-19. URL disponible en: https://archive.org/stream/biostor-102526/ biostor-102526 djvu.txt

Sobrevila C. 1992. Evaluación ecológica rápida. Programa de Ciencias para América Latina. Arlington: The Nature Conservancy; $232 \mathrm{pp}$.

Tirira D. 2000. Listado bibliográfico sobre los mamíferos del Ecuador. Boletines bibliográficos sobre la biodiversidad del Ecuador 2. Publicación especial sobre los mamíferos del Ecuador 3. Quito: EcoCiencia/SIMBIOE; 354 pp. URL disponible en: http://digitalrepository. unm.edu/cgi/viewcontent.cgi?article $=1469 \&$ context=abya yala

UICN. 2013. The UICN red list of threatened species. URL disponible en: http://www.iucnredlist.org/

Voss RS, Emmons LH. 1996. Mammalian diversity in Neotropical lowland rainforests: a preliminary assessment. Bull Am Mus Nat Hist. 230: 1-120. URL disponible en: http://digitallibrary.amnh.org/bitstream/ handle/2246/1671//v2/dspace/ingest/pdfSource/bul/ B230a01.pdf? sequence $=1 \&$ isAllowed $=\mathrm{y}$ 